Research paper

\title{
Variability estimation in resistive switching devices, a numerical and kinetic Monte Carlo perspective
}

\author{
D. Maldonado ${ }^{\text {a }}$, S. Aldana ${ }^{\text {a }}$ M.B. González ${ }^{\mathrm{b}}$, F. Jiménez-Molinos ${ }^{\mathrm{a}}$, M.J. Ibáñez ${ }^{\mathrm{c}}$, D. Barrera $^{\mathrm{c}}$, \\ F. Campabadal ${ }^{\mathrm{b}}$, J.B. Roldán ${ }^{\mathrm{a}, *}$ \\ ${ }^{a}$ Departamento de Electrónica y Tecnología de Computadores, Universidad de Granada, Facultad de Ciencias, Avd. Fuentenueva s/n, 18071, Granada, Spain \\ ${ }^{\mathrm{b}}$ Institut de Microelectrònica de Barcelona, IMB-CNM (CSIC), Carrer dels Til.lers, s/n. Campus UAB, 08193 Bellaterra, Spain \\ ${ }^{\mathrm{c}}$ Departamento de Matemática Aplicada, Universidad de Granada, Facultad de Ciencias, Avd. Fuentenueva s/n, 18071 Granada, Spain
}

\section{A R T I C L E I N F O}

\section{Keywords:}

Resistive switching memory

RRAM

Parameter extraction

Kinetic Monte Carlo simulation

Variability

Modeling

Numerical techniques

\begin{abstract}
A B S T R A C T
We have analyzed variability in resistive memories (Resistive Random Access Memories, RRAMs) making use of advanced numerical techniques to process experimental measurements and simulations based on the kinetic Monte Carlo technique. The devices employed in the study were fabricated using the TiN/Ti/ $/ \mathrm{HfO}_{2} / \mathrm{W}$ stack. The switching parameters were obtained making use of new developed extraction methods. The appropriateness of the advanced parameter extraction methodologies has been checked by comparison to kinetic Monte Carlo simulations; in particular, the reset and set events have been studied and detected. The data obtained were employed to shed light on the resistive switching operation and the cycle-to-cycle variability. It has been shown that variability depends on the numerical technique employed to obtain the set and reset voltages, therefore, this issue must be taken into consideration in RS characterization and modeling studies. The proposed techniques are complementary and depending on the technology and the curves shape the features of a particular method could make it to be the most appropriate.
\end{abstract}

\section{Introduction}

Resistive switching devices have shown great potential to become the key electron devices in the next generation of embedded non-volatile memories circuits [1]. These devices, which are a subgroup of a greater set known as memristors [2], show promising characteristics such as low power operation, good endurance [3] and retention, CMOS technology compatibility, fast write/read times, etc. [1,4-8]. For these reasons, they are considered good candidates for embedded storage class memory in future ICs.

Another booming application where resistive switching devices are called to play an important role is linked to neuromorphic computing. These devices can mimic biological synapses in order to simplify the fabrication of hardware neural networks to build artificial intelligence accelerators [9-22]. Neuromorphic circuits have greatly evolved since the first designs proposed by Carver Mead [23]; in recent years, crossbar arrays made of resistive switching devices have shown the way to implement vector-matrix multiplication, a key module both for the training and inference functions in hardware neural networks. In addition to the scaling and low power operation possibilities related to resistive switching devices in the neuromorphic landscape, it has been reported how their inherent variability can be used to improve commonly found hurdles in machine learning, such as overfitting $[18,24]$.

A third application field for this technology, connected to the natural stochasticity of these devices [1,5,25-27], is associated with the implementation of security modules in hardware devoted to cryptography [27-30]. This facet of the electronic circuits is growing rapidly due to the demands of edge devices for secure data analysis and transmission on the Internet of Things.

Among the features that allow the maturity discernment of a technology, we can count the existence of reliable and flexible compact models. These models along with the accompanying parameter extraction techniques are one of the main pillars of Electronic Design Automation EDA tools. Concerning RRAM models, there have been many works on the last few years [5,31-38]; nevertheless, the parameter extraction facet, a difficult issue in these devices, has not been tackled so often. We deal with this latter issue here focusing on the mathematical

\footnotetext{
* Corresponding author.

E-mail address: jroldan@ugr.es (J.B. Roldán).
} 


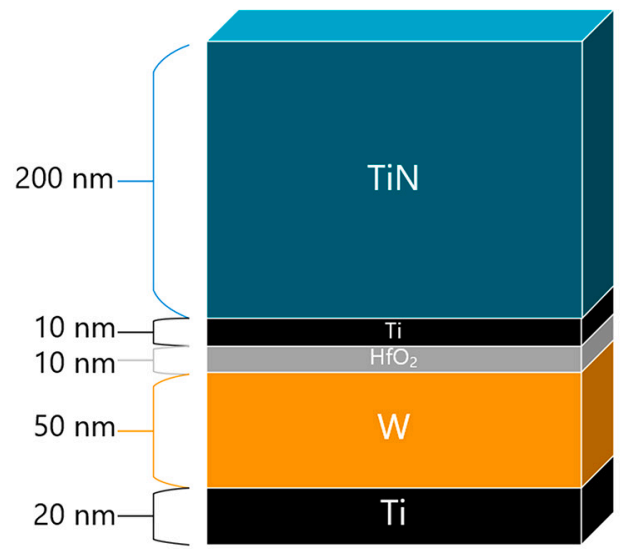

(a)

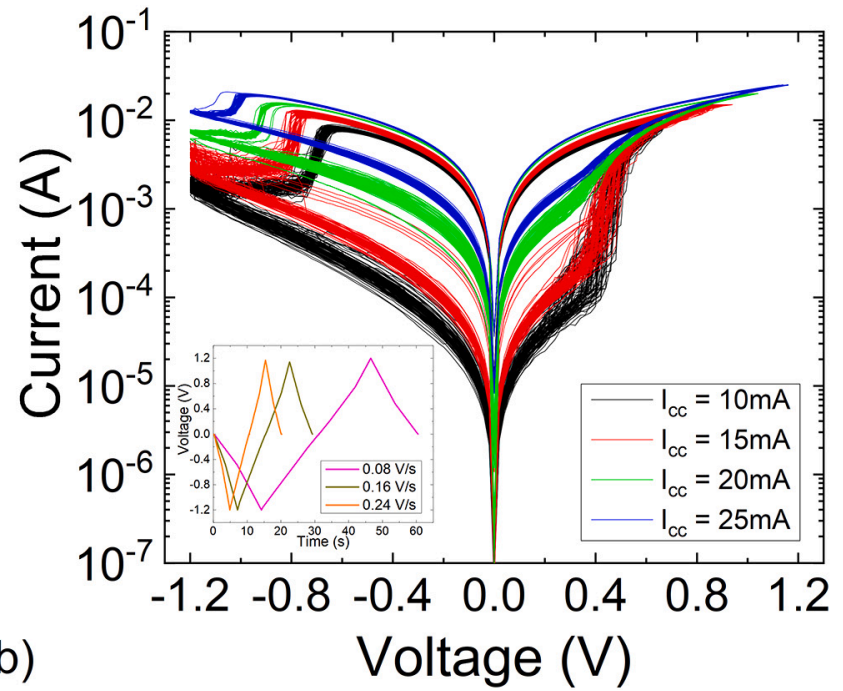

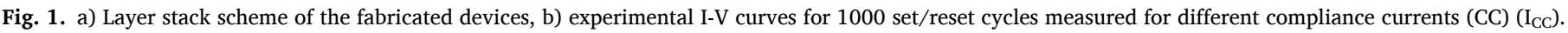

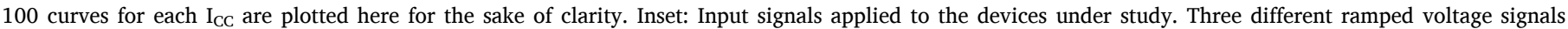
were considered.

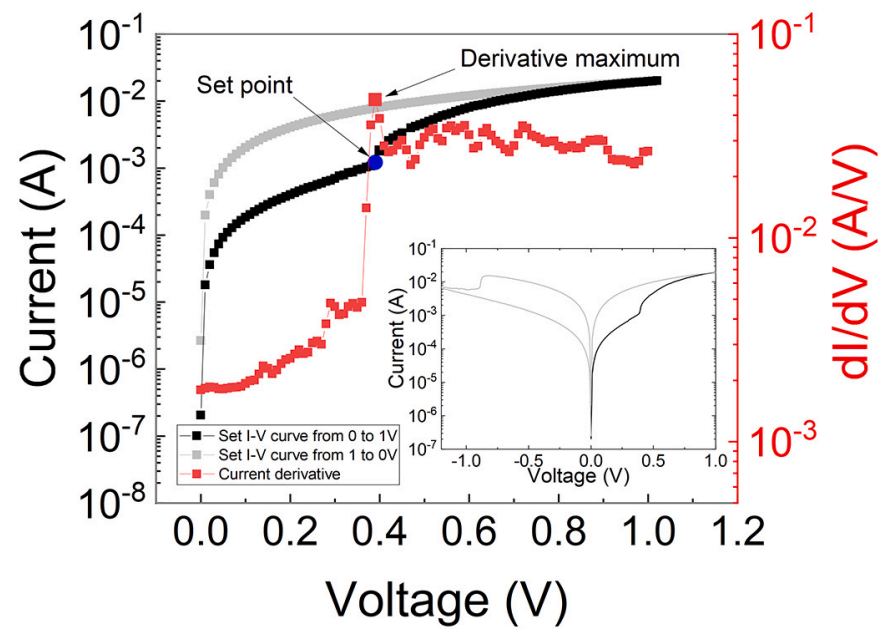

Fig. 2. Experimental set current (black symbols) and first derivative (red symbols) versus voltage. The set point is established (blue point) at the current derivative maximum. We include in the inset a whole set (positive voltage) and reset (negative voltage) cycle to highlight in which part of the curve we are working. (For interpretation of the references to colour in this figure legend, the reader is referred to the web version of this article.)

implementation to build routines to attack the automatic extraction process of thousands of curves at a time, since this is a common situation when dealing with long resistive switching series (to study cycle-to-cycle variability). Moreover, to shed light on the purely numerical procedures that lay behind the extraction process, we have also incorporated comprehensive and physically-based kinetic Monte Carlo (kMC) simulations. In doing so, we pursue the analysis of the step-by-step evolution of conductive filaments (CF) along the device set and reset processes [39-44], taking into consideration that our devices show filamentary conduction [45]. We deepen on the physical mechanisms that control the formation and rupture of percolation paths (the conductive filaments) within a microscopic simulation that includes the 3D solution of the thermal and Poisson equations along with the RS dynamics described by a kinetic Monte Carlo algorithm [39,41].

In Section 2 we introduce the device fabrication and measurement details. Section 3 is devoted to kMC simulation, the numerical techniques employed for the parameter extraction are tackled in Section 4.

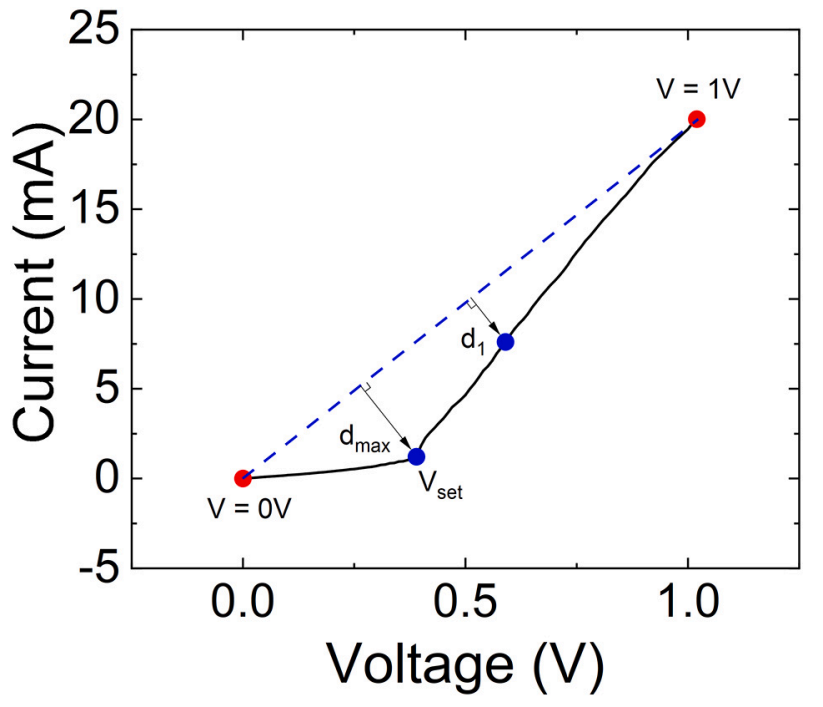

Fig. 3. Experimental set current versus voltage. The new methodology uses a straight line (dashed) to join the final points in the experimental curve, and the maximum distance marks the set voltage.

Section 5 presents and discuss the main results obtained and, finally, the main conclusions are drawn in Section 6.

\section{Device description and measurement}

The TiN/Ti/ $\mathrm{HfO}_{2} / \mathrm{W}$ RRAMs used in this work were fabricated on a highly-doped N-type $(\rho=4 \mathrm{~m} \Omega \cdot \mathrm{cm})$ silicon wafer. The top metal electrode consists of a (200 nm TiN/10 nm Ti) bi-layer and the $50 \mathrm{~nm}$-thick $\mathrm{W}$ bottom electrode was deposited on a $20 \mathrm{~nm}$-thick Ti layer adherence layer on the silicon substrate. The electrical contact to the $\mathrm{W}$ layer is made by Al-metallizing the back of the Si wafer, and the $10 \mathrm{~nm}$-thick $\mathrm{HfO}_{2}$ dielectric layer was grown by ALD [45], see Fig. 1a.

The compliance current $\left(\mathrm{I}_{\mathrm{CC}}\right.$ ) was fixed to $10 \mathrm{~mA}, 15 \mathrm{~mA}, 20 \mathrm{~mA}$ and $25 \mathrm{~mA}$ for different groups of I-V curves, where complete reset and set processes were obtained in long RS series (1000 cycles each) under ramped voltage stress (RVS). We used ramps of different slopes. The colour code for these curves with respect to $\mathrm{I}_{\mathrm{CC}}$, as shown in Fig. 1, is 

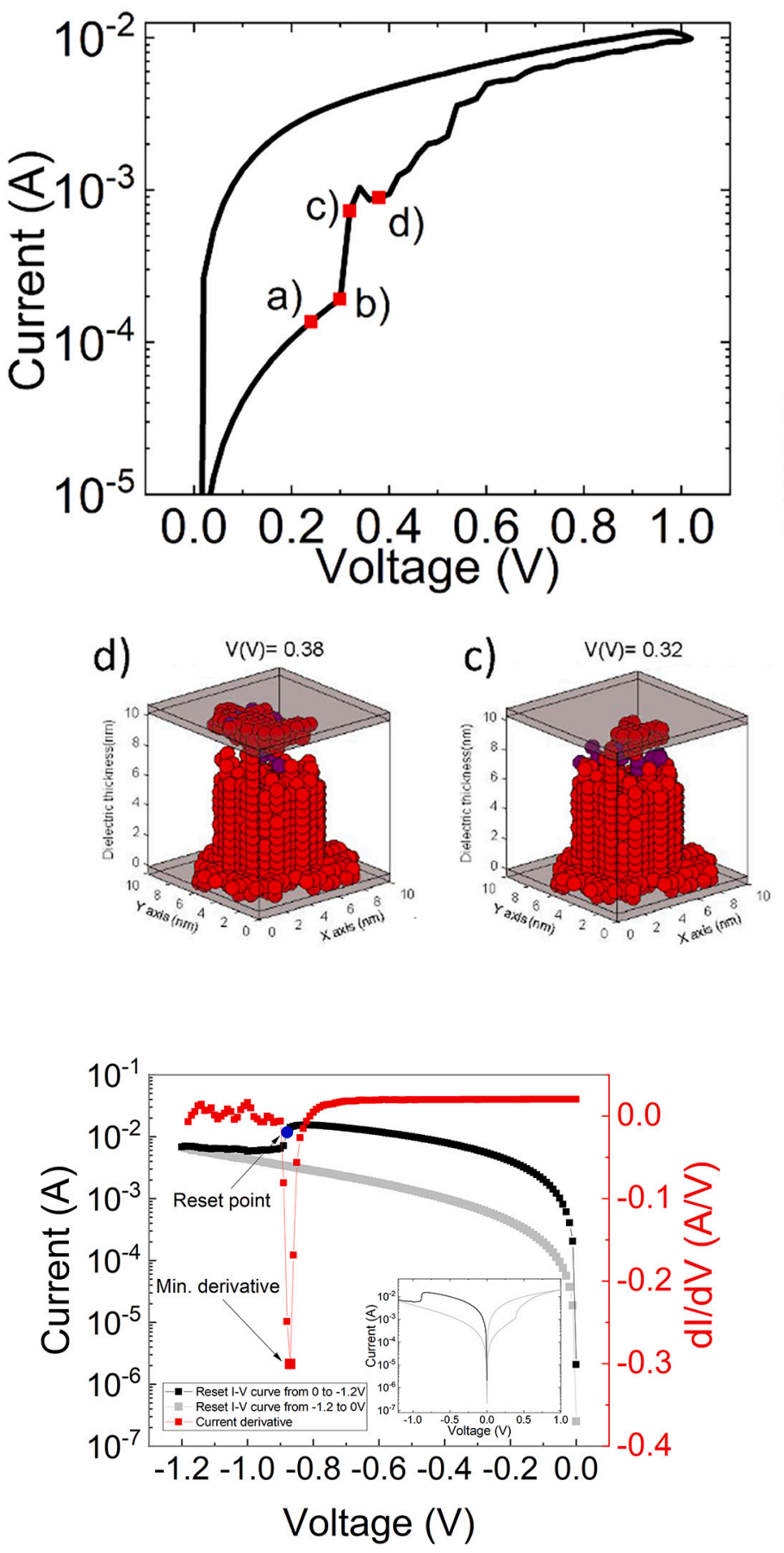

Fig. 5. Experimental reset current (black symbols) and first current derivative (red symbols) versus voltage for the devices under study. The reset point is established by determining the minimum current derivative. (For interpretation of the references to colour in this figure legend, the reader is referred to the web version of this article.)

used henceforth. Since the time step was fixed for the voltage ramps, we just change the voltage step $(\Delta \mathrm{V})$ in the sweeps to obtain different slopes, (see the inset in Fig. 1b). In particular, we considered the following voltage steps: $\Delta \mathrm{V}=0.01 \mathrm{~V}, 0.02 \mathrm{~V}$ and $0.03 \mathrm{~V}$, combined with the compliance currents reported above. The input voltage signal was applied to the TiN/Ti top electrode, while the bottom electrode was grounded. The RS cycles were programmed with a Matlab software tool that controls a Keysight B1500A via GPIB.

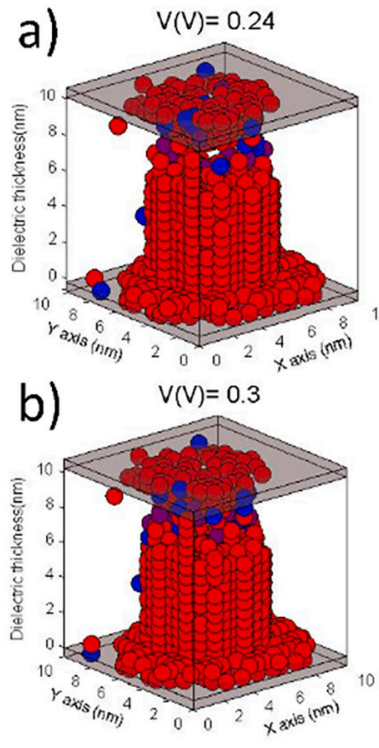

Fig. 4. Kinetic Monte Carlo simulated I-V curve for a set process in a structure similar to the devices under study. a-d) show the oxygen vacancy configuration at four different points along the set curve close to the percolation path formation voltage. Blue particles represent oxygen ions, red particles stand for oxygen vacancies and purple particles are grid points where an oxygen ion and a vacancy coexist [39], but the recombination event has not yet taken place. The different conductive filament snap-shots show the progressive CF creation until the percolation path is fully formed. a) and b), these figures show a simulation point previous to the CF complete formation, c) the simulation point where the percolation path is already formed and d) stands for a consolidated fully formed CF. Kinetic Monte Carlo simulations are good at reproducing the inherent cycle-to-cycle variability of RS devices [39]. (For interpretation of the references to colour in this figure legend, the reader is referred to the web version of this article.)

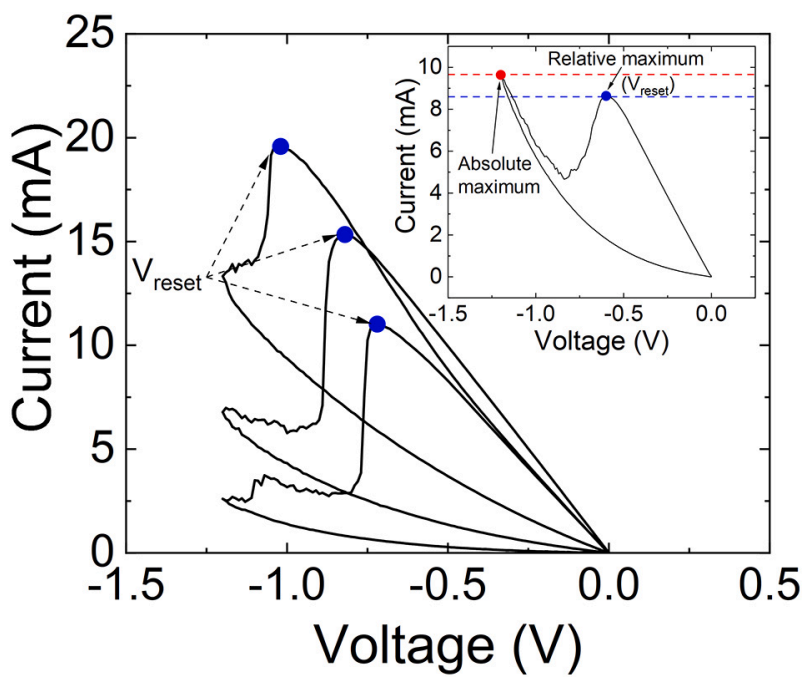

Fig. 6. Experimental reset current versus voltage curves for the devices under study. The maximum value of the curve was established as the reset point. The inset shows an experimental reset current versus voltage, in this case, the current maximum could lead us to a wrong result.

\section{Kinetic Monte Carlo simulation}

A 3D kinetic Monte Carlo simulator was employed to analyze set and reset curves in the structures described in the previous section. The details of this tool have been given elsewhere [39,41]. The Poisson and heat equations are solved for each time step to determine the $3 \mathrm{D}$ temperature and electric field distributions, which are needed to calculate 

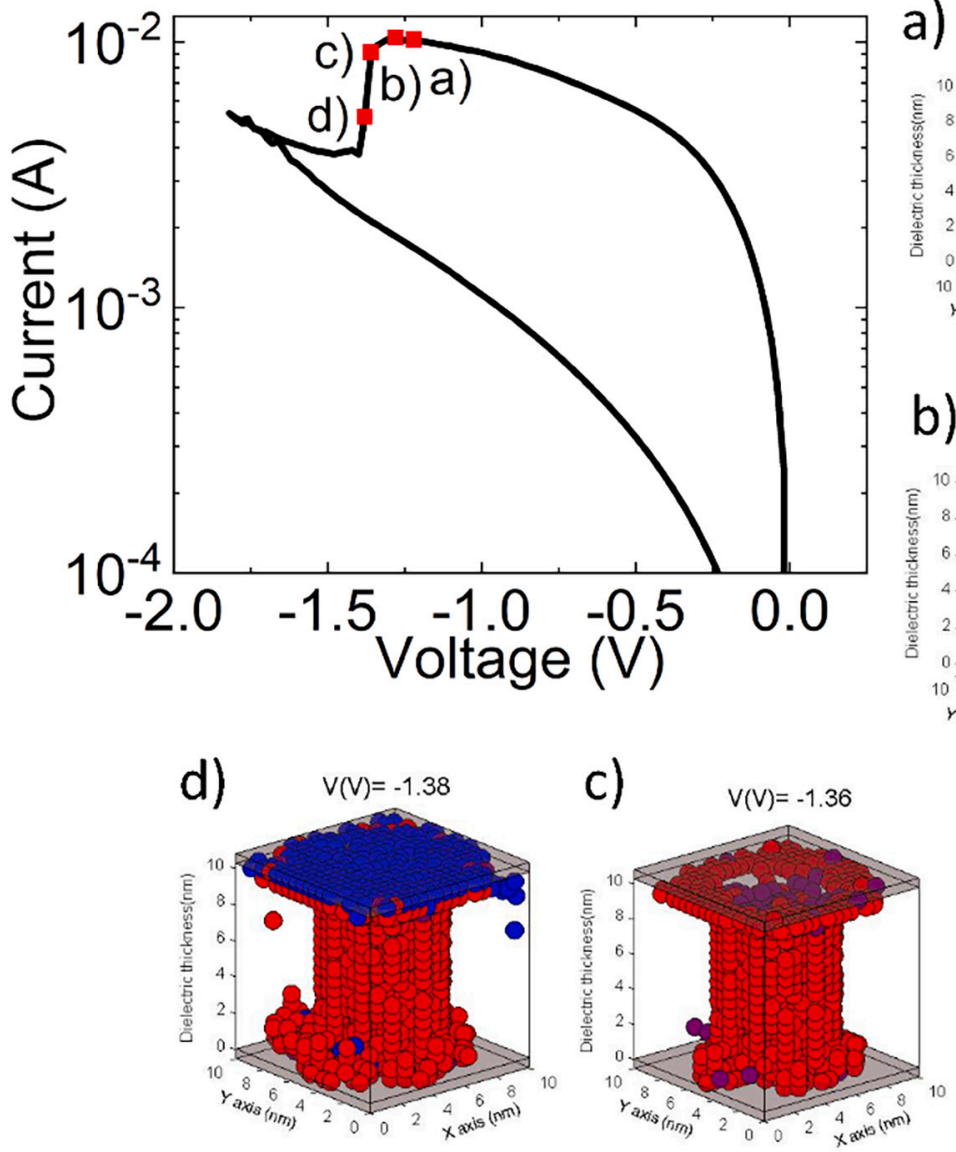
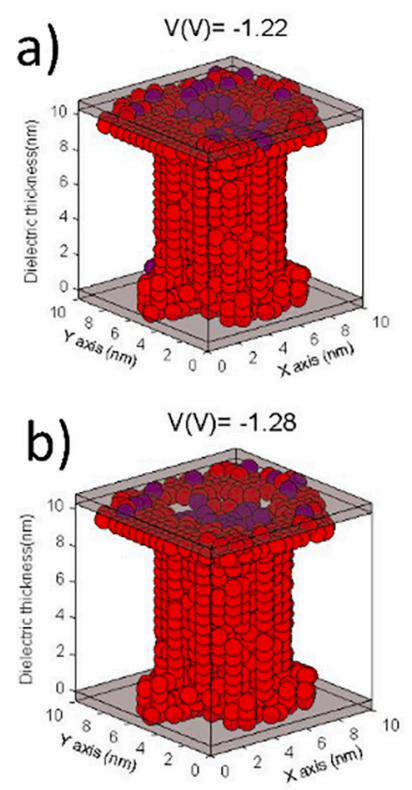

Fig. 7. Kinetic Monte Carlo simulated I-V curve for a structure similar to the devices under study. a-d), the figures show the atomic configuration at four different points along the reset curve near the rupture point. Blue particles are oxygen ions, red particles are oxygen vacancies and purple particles are grid points where an oxygen ion and a vacancy coexist before their recombination. Figure c) shows the simulation point previous to the percolation path rupture and d) the simulation point after the rupture. (For interpretation of the references to colour in this figure legend, the reader is referred to the web version of this article.) the different mechanisms involved in the CF formation and destruction. These mechanisms are randomly activated using a kMC algorithm based on the Transition State Theory [46]. The kMC simulation is a suitable tool for studying the $\mathrm{CF}$ rupture processes since it reproduces well the device inherent stochasticity that lies behind the RS cycle-to-cycle variability. The activation energies employed to calculate the transitions rates of the different physical mechanisms were given in Ref. [39].

\section{Numerical techniques for parameter extraction}

We present here several numerical techniques to extract the set and reset voltages and currents in the long RS series reported above. It is important to notice that although the thresholds of RS phenomena are reasonably distinguishable in a single I-V curve by eye (see Fig. 1), the main point in the algorithms described below consists in the implementation of robust and stable numerical procedures to deal with hundreds of curves automatically. The numerical procedures should obtain good results accounting for the inherent device cycle-to-cycle variability that is reflected on the measurements and it is typical of these devices $[1,5-7,39]$.

\subsection{Set voltage determination}

\subsubsection{Method 1 for Set (MS1). Determination of the current derivative maximum}

The first method to determine the set voltage consists in finding the maximum value of the numerical derivative. The determination of the numerical derivative is not an easy issue due to measurement fluctuations among other considerations, in our case a 5-point numerical calculus was performed, as displayed in Eq. (1) to account for the finite difference approximation to the first derivative with excellent results; nevertheless, if the numerical noise was high, a more complex methodology was implemented [47]. It has to be considered that the measurement conditions can complicate this calculation, for instance, high current variations might occur in these devices under the influence of external fields [48] and high temperatures; in these cases, other numerical approaches can do better $[47,49,50]$. Around the derivative maximum (see Fig. 2), a change in the I-V curve slope is produced; therefore, a variation in the charge transport regime is consequently expected that might be linked to the $\mathrm{CF}$ formation stage.

$f^{\prime}(x) \approx \frac{f(x-2 h)-8 f(x-h)+8 f(x+h)-f(x+2 h)}{12 h}$

Once the current derivative has been calculated its maximum can be easily detected, see Fig. 2.

4.1.2. Method 2 for Set (MS2). Maximum separation from a straight line that joins the end points in a set curve

Another methodology that can be implemented to determine the set voltage, $\mathrm{V}_{\text {set }}$, consists in finding the maximum separation of the measured curve to an imaginary straight line that joins the first and end points of the measured curve (see Fig. 3). In other words, it would consist in finding the set curve knee, as shown in Fig. 3 [51]. The original $\mathrm{I}-\mathrm{V}$ curve is plotted from the beginning of the measurement $\left(\mathrm{V}_{\text {Applied }}=0\right.$ $\mathrm{V}$ ) until the end of the voltage ramp (at $\mathrm{V}_{\text {Applied }}=1 \mathrm{~V}$ ). Then, a straight line joins these two points in the I-V domain (dashed line), the furthest point to the measured I-V curve (the greater distance, $d_{\text {max }}$ ) marks the set voltage $\mathrm{V}_{\text {set }}$.

A deeper insight into what is going on in a set process for a particular technology can be seen using a kinetic Monte Carlo simulator [39,41]. In this respect, the $3 \mathrm{D}$ temperature and electric field distributions, and the conductive filament formation steps can be studied systematically to 

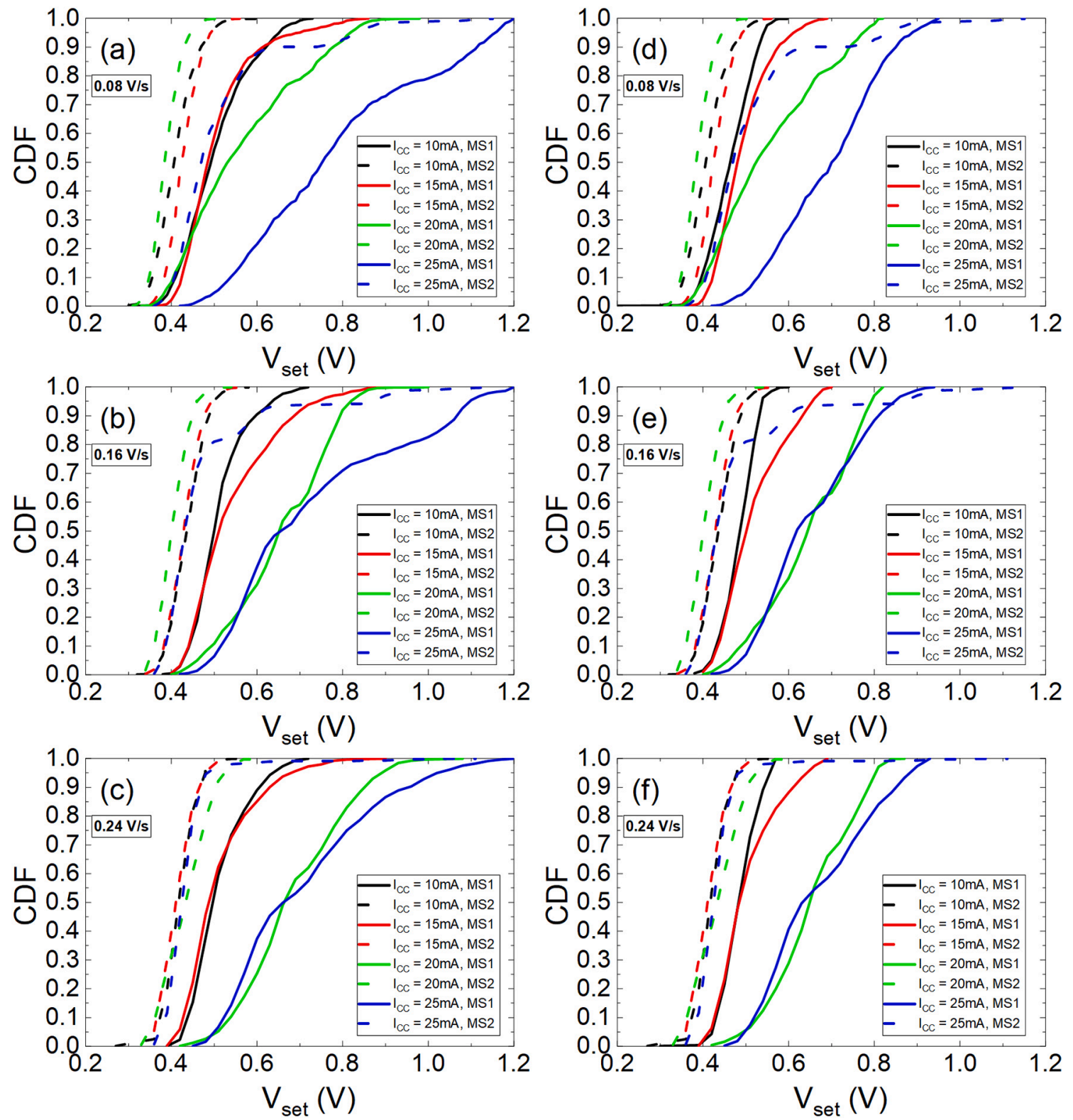

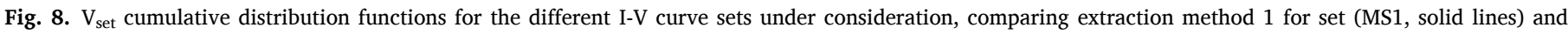

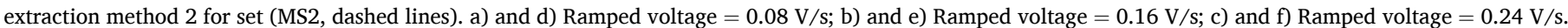

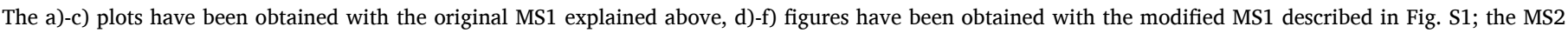
method is the same in all the plots, it is kept for comparison.

shed light on the threshold between the high resistance state (HRS) and the low resistance state (LRS), see Fig. 4.

In Fig. 4, the progressiveness of the $\mathrm{CF}$ formation process is depicted. The simulated curve does not compare in shape with the experimental data in the region close to the set voltage determination, which corresponds to the onset of a change of conduction regime (see the curve portion where point $\mathrm{b}-\mathrm{d}$ are placed). This is due to the discreteness and limitations of the simulation domain, the percolation path searching algorithm, etc. However, it can be seen that in this case the CF full formation and the corresponding change in the device conductivity are close to the slope change region in the set I-V curve. In this respect, the physical foundation of the two proposed numerical methodologies is well established. The first method might provide more accurate results due to its very own definition and connection with the physics underlying the set process; however, other issues have to be considered, as it will be shown below.

\subsection{Reset voltage determination}

4.2.1. Method 1 for Reset (MR1). Determination of the current derivative minimum

The first method to determine the reset voltage consists in finding the current derivative minimum. This procedure is somewhat linked to the first method introduced for the set voltage determination, the numerical derivative is calculated the same way described above. The peak of the minimum value of the current derivative is easily detected numerically, see the red curve in Fig. 5.

\subsubsection{Method 2 for Reset (MR2). Determination of the current maximum}

Another method to determine the reset voltage is based on the calculation of the current maximum, see Fig. 6 .

Depending on the compliance current and the measurement technique, this methodology could show problems related to the location of the current maximum. If the current compliance value is high enough 

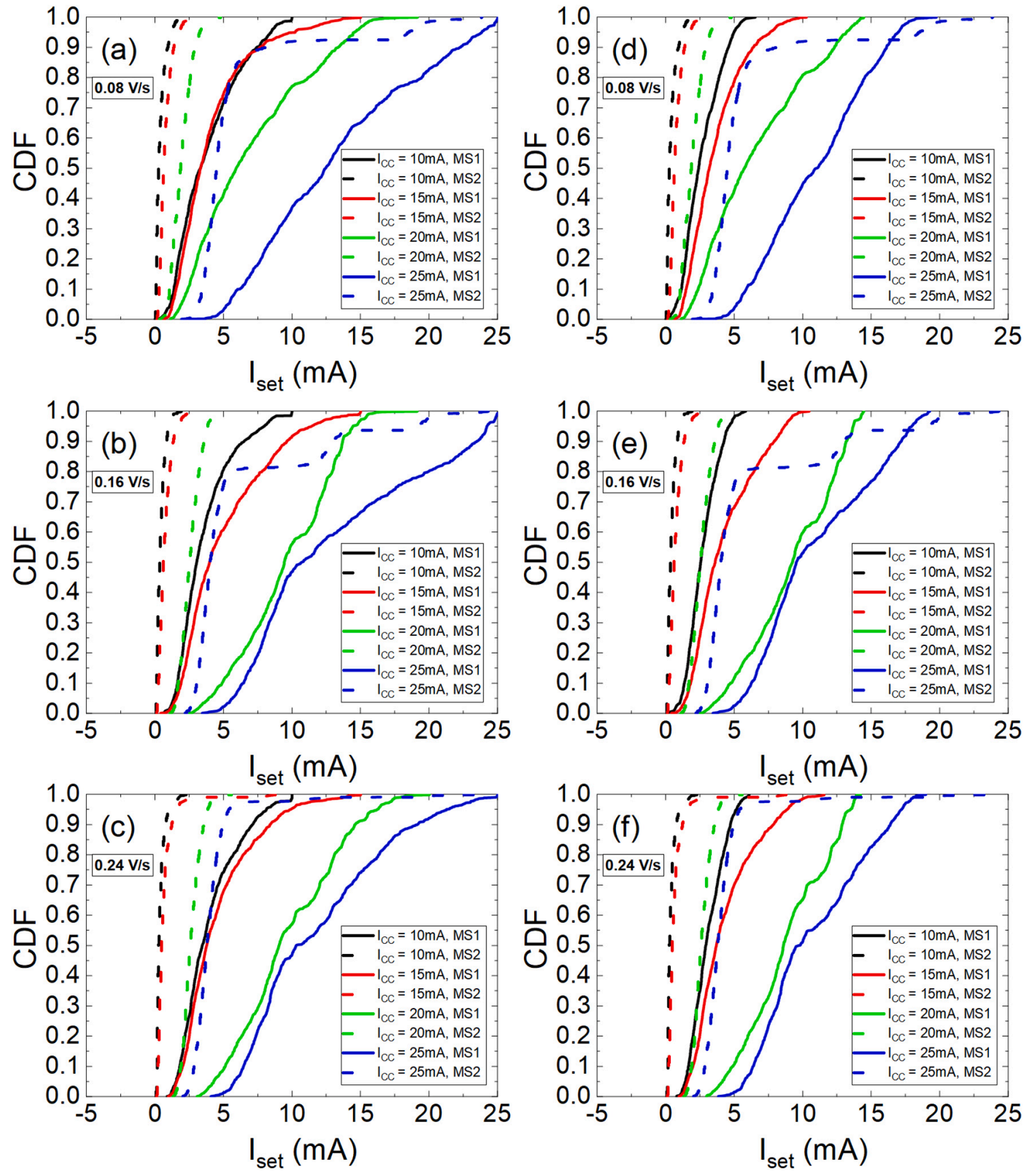

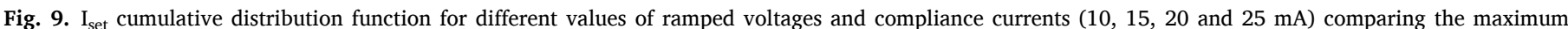

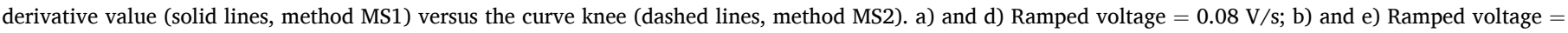
$0.16 \mathrm{~V} / \mathrm{s}$; c) and f) Ramped voltage $=0.24 \mathrm{~V} / \mathrm{s}$. The d)-f) figures have been obtained with the modified MS1 explained in Fig. S1.

(see the inset in Fig. 6) the maximum could be found in the last measured current value in the curve. This occurrence has to be detected and corrected in the algorithm to robustly analyze hundreds of I-V curves.

A kinetic Monte Carlo simulation was also performed in this case. A closer look at the step-by-step CF rupture in the region close to the reset can help to evaluate the location of the percolation path rupture event.

Fig. 7 shows how the rupture point does not necessarily coincide with the maximum current point. Further CF degradation takes place after these maxima are achieved. In addition, it is seen that the final percolation path gets broken somewhere in the curve sections where the current strongest reduction is found. Although the random nature of the physics behind RS could place the percolation path rupture around points c) and d) (this stochasticity is correctly captured by the kMC technique) in other cases, it seems that the first methodology might be closer to the real $\mathrm{CF}$ rupture.

We call the reader's attention to the fact that although these numerical techniques can be applied with generality, other RRAM technologies with different particularities linked to particular materials or interfaces and fabrication techniques could require adaptations to improve the parameter extraction procedures.

\section{Results and discussion}

We have employed the methodologies described previously to extract the most representative RS parameters $\left(V_{\text {set }}, I_{\text {set }}, V_{\text {reset }}\right.$ and $\left.I_{\text {reset }}\right)$ in the different series of cycles measured. As commented above, different ramped input voltages and compliance currents were considered. The $\mathrm{V}_{\text {set }}$ cumulative distribution functions (CDF) are given in 


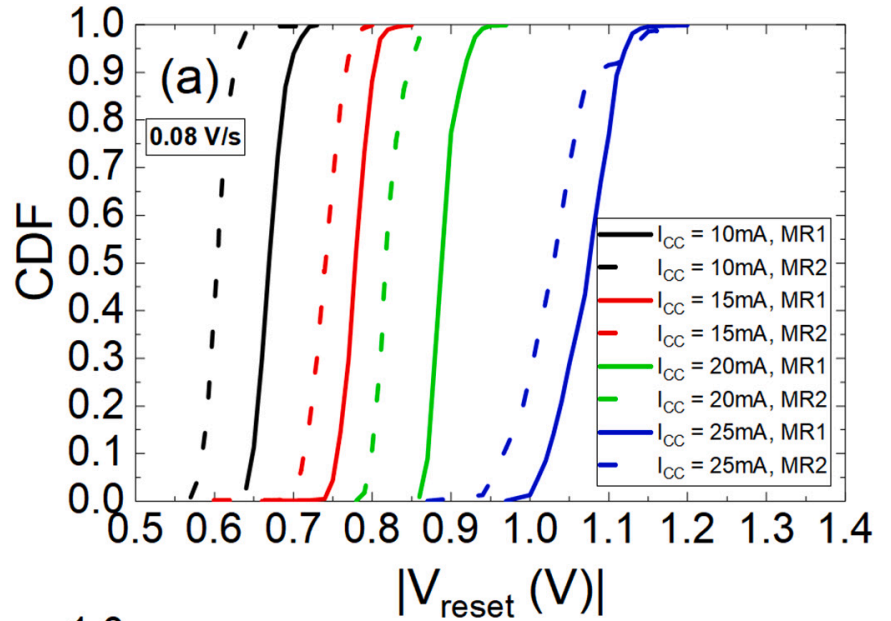

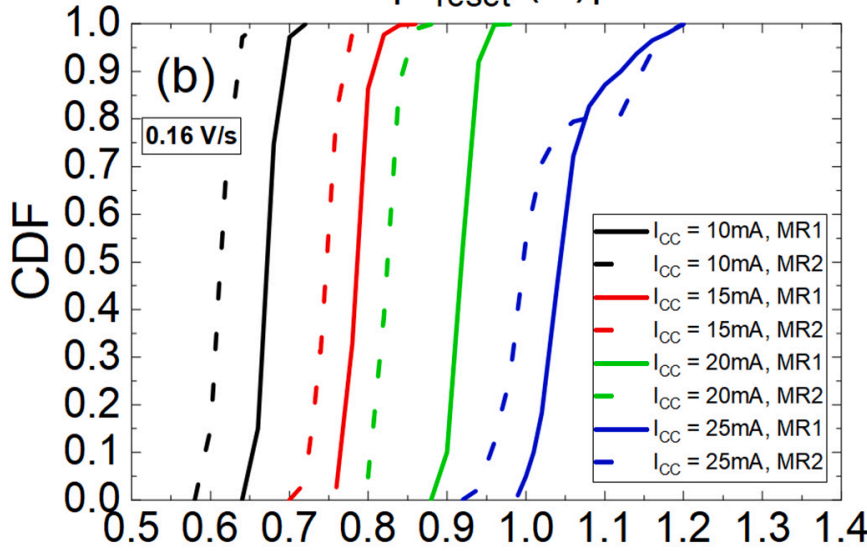

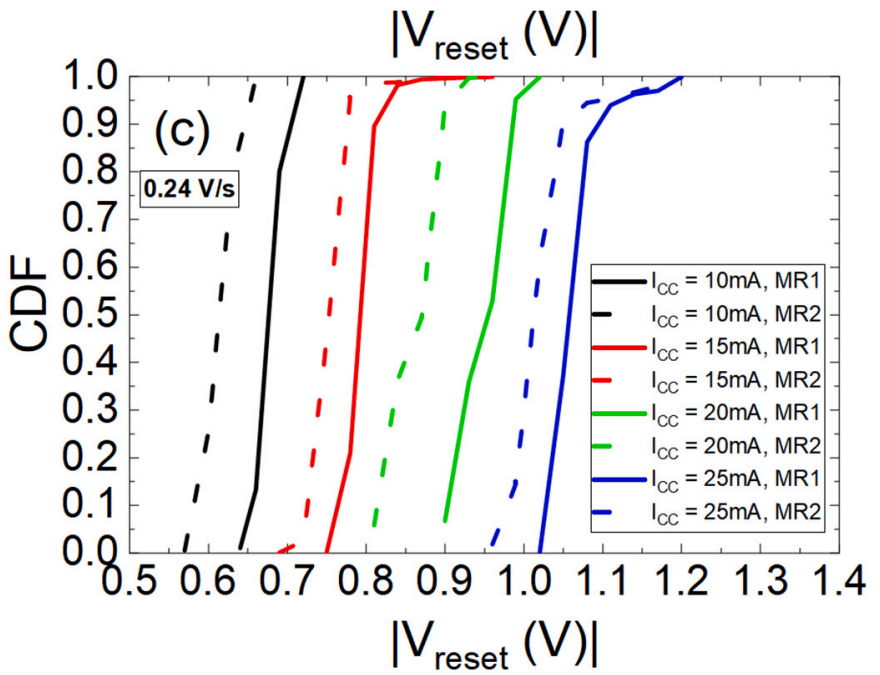

Fig. 10. $\mathrm{V}_{\text {reset }}$ cumulative distribution functions for different values of ramped voltages and compliance currents $(10,15,20$ and $25 \mathrm{~mA})$ comparing the maximum derivative value (solid lines, MR1) versus the maximum current (dashed lines, MR2). a) Ramped voltage $=0.08 \mathrm{~V} / \mathrm{s}$, b) Ramped voltage $=0.16$ $\mathrm{V} / \mathrm{s}$, c) Ramped voltage $=0.24 \mathrm{~V} / \mathrm{s}$.

Fig. 8a-c.

As can be seen, the curve knee determination (MS2) provides lower set voltages than the current derivative maximum method (MS1). In addition, the variability described by the CDFs is much different. See that in Fig. 8a, b, d and e, for an $\mathrm{I}_{\mathrm{CC}}=20 \mathrm{~mA}$ (MS2) we obtain lower voltages compared to other compliance currents. These results could be feasible due to a different CF shape for this RS series and the role of virtual electrodes [39]; effects linked to numerical issues could also be
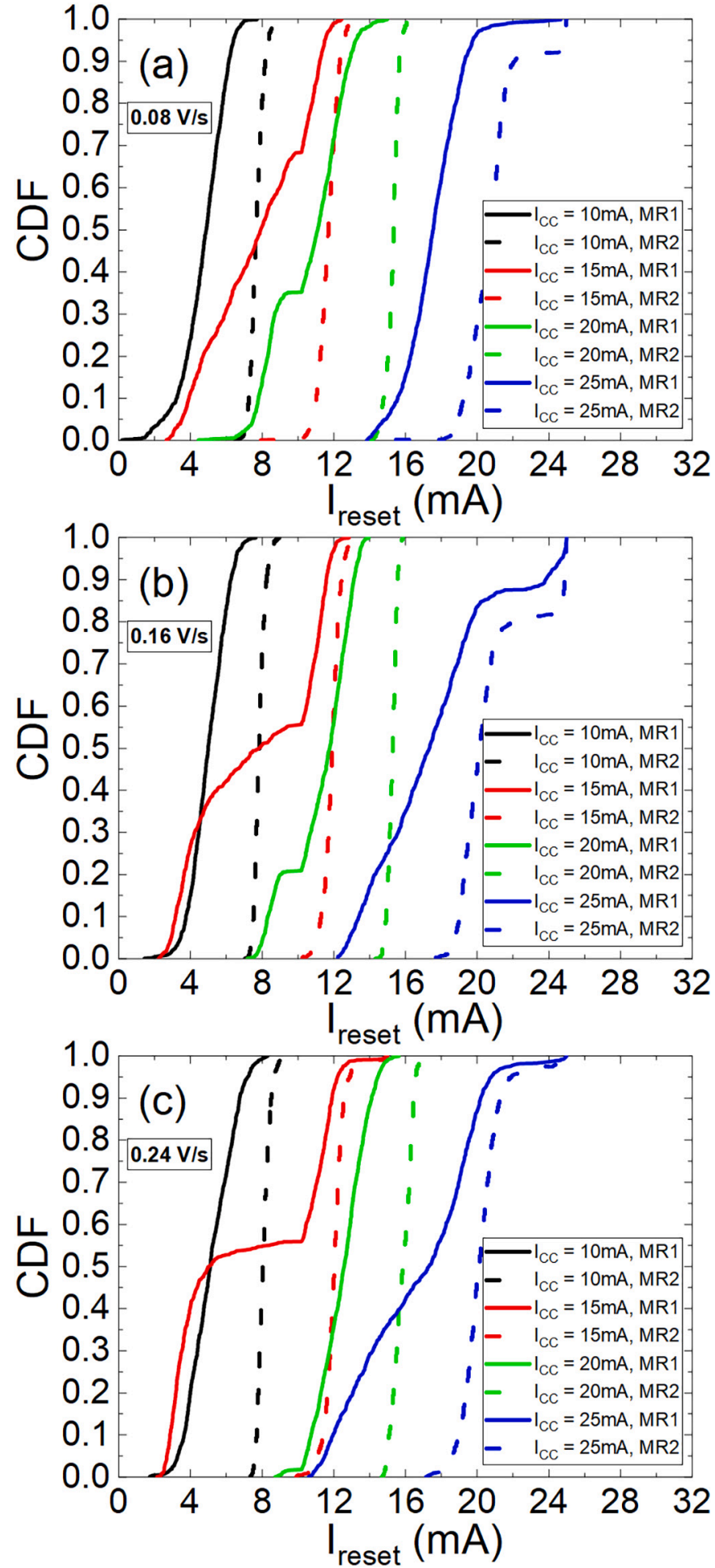

Fig. 11. $I_{\text {reset }}$ cumulative distribution functions for different values of ramped voltages and compliance currents $(10,15,20$ and $25 \mathrm{~mA})$ comparing the maximum derivative value (solid lines, MR1) versus the maximum current (dashed lines, MR2). a) Ramped voltage $=0.08 \mathrm{~V} / \mathrm{s}$, b) Ramped voltage $=0.16$ $\mathrm{V} / \mathrm{s}$, c) Ramped voltage $=0.24 \mathrm{~V} / \mathrm{s}$.

behind this issue. Although a higher current is allowed that rupture could be placed at lower voltages due to a $\mathrm{CF}$ narrowing. This is an important issue since many authors evaluate cycle-to-cycle variability by analyzing the set and reset voltage CDFs; it is clear (as shown in Fig. 8a-c) that the CDF shape depends on the algorithm employed to extract these parameters. Therefore, the variability evaluation has to be 

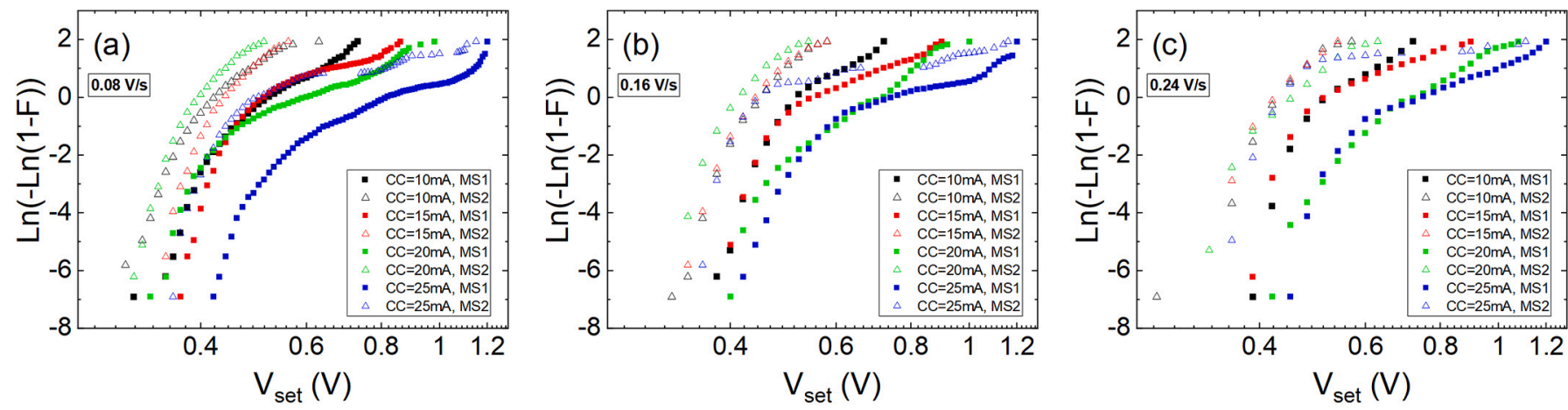

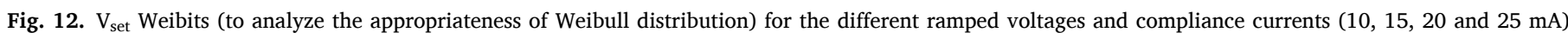
described in Section 2. a) Ramped voltage $=0.08 \mathrm{~V} / \mathrm{s}$, b) Ramped voltage $=0.16 \mathrm{~V} / \mathrm{s}, \mathrm{c}$ ) Ramped voltage $=0.24 \mathrm{~V} / \mathrm{s}$.
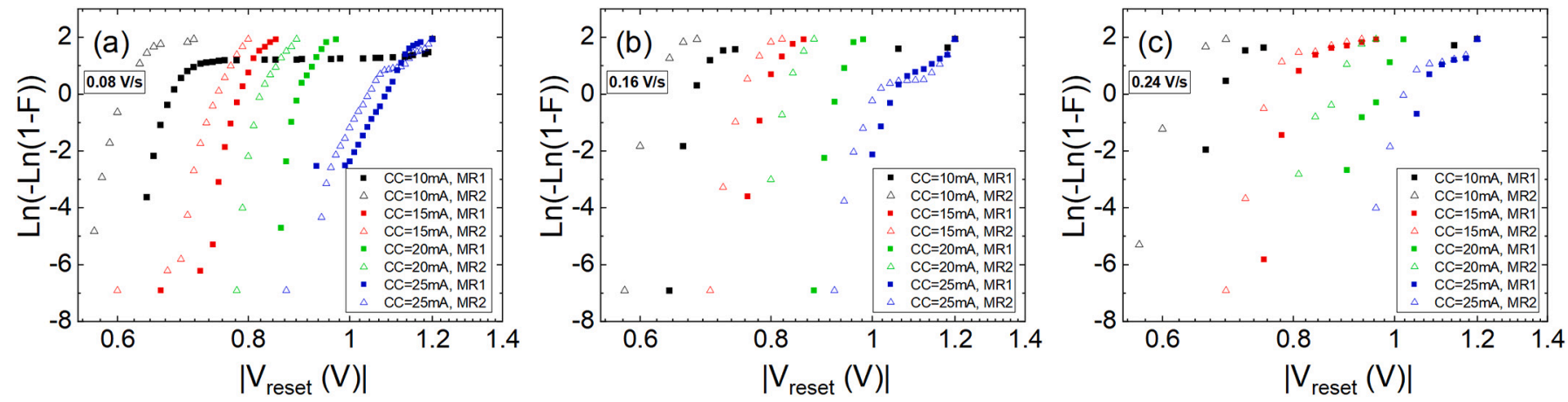

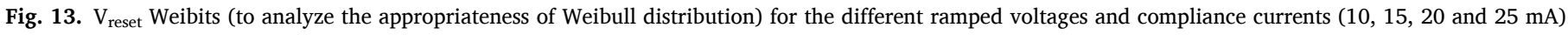
described in Section 2. a) Ramped voltage $=0.08 \mathrm{~V} / \mathrm{s}$, b) Ramped voltage $=0.16 \mathrm{~V} / \mathrm{s}$, c) Ramped voltage $=0.24 \mathrm{~V} / \mathrm{s}$.

considered with care. It can be also noticed that extraction methodologies based on the numerical derivative (MS1 in our case) are dependent on measurement noise $[47,49,50]$. This fact could lead to error in nearly constant slope curves, see the supplementary material (Fig. S1). In our case, to improve the extraction methodology, we have limited the voltage interval where MS1 is applied as explained in the supplementary material. The results of the new MS1 technique are shown in Fig. 8d, e, $\mathrm{f}$ along with the same results for MS2 shown in Fig. 8a-c.

The same tendencies reported above can be observed in Fig. 9d-e, for $I_{\text {set }}$ case, where we have also considered the results of the modified MS1. This parameter is directly extracted since it is the current that corresponds to the set voltage in the I-V curve.

The results obtained for method 2, again, show lower currents and lower variability. See that, as expected, the set currents increase with the compliance current level despite the ramped voltage.

For the $\mathrm{V}_{\text {reset }}$ (Fig. 10) and $\mathrm{I}_{\text {reset }}$ (Fig. 11), the results show a lower spreading than in the set case for both extraction methodologies. Both reset methods deal with an easier determination process due to the curves shape.

As expected, higher reset voltages and currents are obtained for greater current compliances. Variability is much lower than in the set case, in general; it is also worth mentioning that for method 2 (current maximum) there is a lower variability than in method 1 . Other methodologies have been proposed for the reset voltage determination [52] although these definitions were more adequate for the technology analyzed in this reference (for the unipolar devices analyzed there, the I$\mathrm{V}$ curves showed a sudden current drop once the reset voltage was reached). In particular, the reset voltage was defined as the voltage where a certain reduction of current is obtained in two consecutive points $\left(\mathrm{V}_{\text {reset }}=\mathrm{V}_{\mathrm{i}}\right.$ if $\mathrm{I}_{\mathrm{i}+1} \leq(1-\mathrm{a}) \mathrm{I}_{\mathrm{i}}$, where $\left.0<\mathrm{a}<1\right)$. For certain technologies, values of $\mathrm{a}=0.3$ or higher can be employed [52], although in our case, a value below 0.2 has to be used, see the supplementary material (Fig. S2). In this respect, the techniques presented here can be tuned (increasing or decreasing the voltage sweep search interval, or fixing a different value for the parameter devoted to detect a certain current drop) to deal with I-V curves of different shapes connected to other technologies that might show, for instance, progressive set and reset processes.

In Figs. 12 and 13, the Weibits of the $\mathrm{V}_{\text {set }}$ and $\mathrm{V}_{\text {reset }}$ obtained above are plotted. Although the Weibull distribution works well with data generated in systems that show weakest-link reliability behavior (the failure of the whole, in this case, related to the conductive filament formation and rupture, is dominated by the degradation rate for the weakest element $[53,54]$ ); the expected straight line does not show up. The reason behind this is that the RS physics is complex and involves different intertwined mechanisms that when put together do not follow a Weibull distribution. In this respect, Phase type distributions [26,53] achieve a much better fitting, although we do not enter into details of the statistical structure of our data since this is out of the scope of this work.

See in Fig. 13 that the $V_{\text {reset }}$ Weibits show a more linear shape for input signals with lower ramps. As the ramp rate increases the data distribution is spread out. The reasons behind this might be related to different conditions of electric field and temperature, this could lead to more porous conductive filaments that produce a different distribution of reset voltages $[38,39,55]$.

\section{Conclusions}

Different numerical techniques for extracting typical RRAM resistive switching parameters, such as set and reset voltages and currents, have been developed and tested. After automatically processing hundreds of experimental curves obtained in long RS series, the results have been analyzed. To do so, valence change memories have been fabricated and measured. We have made use of cumulative distribution functions and 
the Weibull distribution to analyze the results, assess the cycle-to-cycle variability that is obtained for each of the methodologies presented. The subtleties related to methods based on the processing of the current numerical derivative are described and its connections to results obtained employing kinetic Monte Carlo simulations have been explained. In general, the procedures linked to the current derivative determination present higher variability than the other methods, although the appropriateness of a particular method could depend on the shape of the current-voltage curve. We have found that the evaluation of cycle-tocycle variability depends greatly on the numerical method employed to extract the RS parameters.

Supplementary data to this article can be found online at https://doi. org/10.1016/j.mee.2022.111736.

\section{Author contributions}

Conceptualization, J.B.R., F.C.; software, D.M., D.B., M.J.I., S.A.; data curation, D.M. M.B.G., S.A.; writing - original draft, J.B.R., D.M., review \& editing, J.B.R., F.J.-M., D.M., F.C.

\section{Declaration of Competing Interest}

The authors declare that they have no known competing financial interests or personal relationships that could have appeared to influence the work reported in this paper.

\section{Acknowledgments}

The authors thank the support of the Spanish Ministry of Science, Innovation and Universities and the FEDER program through projects TEC2017-84321-C4-1-R, TEC2017-84321-C4-3-R, and projects A. TIC.117.UGR18, IE2017-5414 and B.TIC.624.UGR20 funded by the Consejería de Conocimiento, Investigación y Universidad, Junta de Andalucía (Spain) and the FEDER program. Funding for open access charge: Universidad de Granada/CBUA

\section{References}

[1] M. Lanza, H.-S.P. Wong, E. Pop, D. Ielmini, D. Strukov, B.C. Regan, L. Larcher, M. A. Villena, J.J. Yang, L. Goux, A. Belmonte, Y. Yang, F.M. Puglisi, J. Kang, B. Magyari-Köpe, E. Yalon, A. Kenyon, M. Buckwell, A. Mehonic, A. Shluger, H. Li, T.-H. Hou, B. Hudec, D. Akinwande, R. Ge, S. Ambrogio, J.B. Roldan, E. Miranda, J. Suñe, K.L. Pey, X. Wu, N. Raghavan, E. Wu, W.D. Lu, G. Navarro, W. Zhang, H. Wu, R. Li, A. Holleitner, U. Wurstbauer, M. Lemme, M. Liu, S. Long, Q. Liu, H. Lv, A. Padovani, P. Pavan, I. Valov, X. Jing, T. Han, K. Zhu, S. Chen, F. Hui, Y. Shi, Recommended methods to study resistive switching devices, Adv. Electron. Mater. 5 (2019) 1800143.

[2] Leon O. Chua, Mo Kang Sung, Memristive devices and systems, Proc. IEEE 64 (2) (1976) 209-223.

[3] M. Lanza, R. Waser, D. Ielmini, J.J. Yang, L. Goux, J. Suñe, A.J. Kenyon, A. Mehonic, S. Spiga, V. Rana, S. Wiefels, S. Menzel, I. Valov, M.A. Villena, E. Miranda, X. Jing, F. Campabadal, M. Gonzalez, F. Aguirre, F. Palumbo, K. Zhu, J. B. Roldan, F.M. Puglisi, L. Larcher, T.-H. Hou, T. Prodromakis, Y. Yang, P. Huang, T. Wang, Y. Chai, K.L. Pey, N. Raghavan, S. Duenas, T. Wang, Q. Xia, S. Pazos, Standards for the characterization of endurance in resistive switching devices, ACS Nano 15 (11) (2021) 17214-17231.

[4] F. Pan, S. Gao, C. Chen, C. Song, F. Zeng, Recent progress in resistive random access memories: materials, switching mechanisms and performance, Mater. Sci. Eng. 83 (2014) 1-59.

[5] D. Ielmini, R. Waser, Resistive Switching: From Fundamentals of Nanoionic Redox Processes to Memristive Device Applications, Wiley-VCH, 2015.

[6] J.S. Lee, S. Lee, T.W. Noh, Resistive switching phenomena: a review of statistical physics approaches, Appl. Phys. Rev. 2 (2015), 031303.

[7] S. Spiga, A. Sebastian, D. Querlioz, B. Rajendran, Memristive Devices for BrainInspired Computing, Elsevier, 2020.

[8] S. Ito, Y. Hayakawa, Z. Wei, S. Muraoka, K. Kawashima, H. Kotani, K. Kouno, M. Nakamura, G.A. Du, J.F. Chen, ReRAM technologies for embedded memory and further applications, in: 2018 IEEE International Memory Workshop (IMW), 2018, pp. $1-4$.

[9] S. Yu, Y. Wu, R. Jeyasingh, D. Kuzum, H.-S. Wong, An electronic synapse device based on metal oxide resistive switching memory for neuromorphic computation, IEEE Trans. Electron. Devices 58 (8) (2011) 2729-2737, https://doi.org/10.1109/ TED.2011.2147791.
[10] F. Hui, P. Liu, S.A. Hodge, T. Carey, C. Wen, F. Torrisi, D. Thanuja, L. Galhena, F. Tomarchio, Y. Lin, E. Moreno, J.B. Roldan, E. Koren, A.C. Ferrari, M. Lanza, Insitu observation of low-power nano-synaptic response in graphene oxide using conductive atomic force microscopy, Small 17 (2021) 2101100.

[11] S. Ambrogio, et al., Equivalent-accuracy accelerated neural-network training using analogue memory, Nature 558 (2018) 60-67.

[12] P.A. Merolla, J.V. Arthur, R. Alvarez-Icaza, A.S. Cassidy, J. Sawada, F. Akopyan, B. L. Jackson, N. Imam, C. Guo, Y. Nakamura, B. Brezzo, I. Vo, S.K. Esser, R. Appuswamy, B. Taba, A. Amir, M.D. Flickner, W.P. Risk, R. Manohar, D. S. Modha, A million spiking-neuron integrated circuit with a scalable communication network and interface, Science 345 (2014) 668-673.

[13] M. Pedro, J. Martin-Martinez, R. Rodriguez, M.B. Gonzalez, F. Campabadal, M. Nafria, An unsupervised and probabilistic approach to Pavlov's dog experiment with OxRAM devices, Microelectron. Eng. 215 (2019), 111024.

[14] F. Alibart, E. Zamanidoost, D.B. Strukov, Pattern classification by memristive crossbar circuits using ex situ and in situ training, Nat. Commun. 4 (2013) 2072.

[15] P. Yao, H. Wu, B. Gao, J. Tang, Q. Zhang, W. Zhang, J.J. Yang, H. Qian, Fully hardware-implemented memristor convolutional neural network, Nature 577 (7792) (2020) 641-646.

[16] M. Prezioso, F. Merrikh-Bayat, B.D. Hoskins, G.C. Adam, K.K. Likharev, D. B. Strukov, Training and operation of an integrated neuromorphic network based on metal-oxide memristors, Nature 521 (2015) 61-64.

[17] M.A. Zidan, J.P. Strachan, W.D. Lu, The future of electronics based on memristive systems, Nature Electronics 1 (2018) 22-29.

[18] R. Romero-Zaliz, E. Perez, F. Jiménez-Molinos, C. Wenger, J.B. Roldán, Study of quantized hardware deep neural networks based on resistive switching devices, conventional versus convolutional approaches, Electronics 10 (2021) 346.

[19] A. Mehonic, D. Joksas, W.H. Ng, M. Buckwell, A.J. Kenyon, Simulation of inference accuracy using realistic RRAM devices, Front. Neurosci. 13 (2019) 593.

[20] A. Sebastian, M. Le Gallo, R. Khaddam-Aljameh, et al., Memory devices and applications for in-memory computing, Nat. Nanotechnol. 15 (2020) 529-544.

[21] D. Sakellaropoulos, P. Bousoulas, G. Nikas, C. Arvanitis, E. Bagakis, D. Tsoukalas, Enhancing the synaptic properties of low-power and forming-free $\mathrm{HfO}_{\mathrm{x}} / \mathrm{TaO}_{\mathrm{y}} / \mathrm{HfO}_{\mathrm{x}}$ resistive switching devices, Microelectron. Eng. 229 (2020), 111358.

[22] S.R. Nandakumar, B. Rajendran, Bio-mimetic synaptic plasticity and learning in a sub-500 mV Cu/SiO2/W, Microelectron. Eng. 226 (2020), p. 111290.

[23] C. Mead, M. Ismail, Analog VLSI Implementation of Neural Systems, Springer, 1989.

[24] T. Dalgaty, N. Castellani, C. Turck, et al., In situ learning using intrinsic memristor variability via Markov chain Monte Carlo sampling, Nat. Electron. 4 (2021) 151-161.

[25] A.N. Mikhaylov, D.V. Guseinov, A.I. Belov, D.S. Korolev, V.A. Shishmakova, M. N. Koryazhkina, D.O. Filatov, O.N. Gorshkov, D. Maldonado, F.J. Alonso, J. B. Roldán, A.V. Krichigin, N.V. Agudov, A.A. Dubkov, A. Carollo, B. Spagnolo, Stochastic Resonance in a Metal-Oxide Memristive Device, Chaos, Solitons \& Fractals, 2021.

[26] J.E. Ruiz-Castro, C. Acal, D. Maldonado, J.B. Roldán, One cut-point phase-type distributions in reliability. An application to resistive random access memories, Mathematics 9 (2021) 2734.

[27] M. Lanza, C. Wen, X. Li, T. Zanotti, F.M. Puglisi, Y. Shi, F. Saiz, A. Antidormi, S. Roche, W. Zheng, X. Liang, J. Hu, S. Duhm, K. Zhu, F. Hui, J.B. Roldan, B. Garrido, T. Wu, V. Chen, E. Pop, Advanced data encryption using twodimensional materials, Adv. Mater. 2100185 (2021) 1-12.

[28] R. Carboni, D. Ielmini, Stochastic memory devices for security and computing, Advanced Electronic Materials 5 (2019) 1900198.

[29] Z. Wei, et al., True random number generator using current difference based on a fractional stochastic model in 40-nm embedded ReRAM, in: 2016 IEEE International Electron Devices Meeting (IEDM), San Francisco, CA, 2016, pp. 4.8.1-4.8.4, https://doi.org/10.1109/IEDM.2016.7838349.

[30] A. Chen, Utilizing the variability of resistive random access memory to implement reconfigurable physical unclonable functions, IEEE Electron Device Lett. 36 (2) (2015) 138-140.

[31] J.B. Roldán, F.J. Alonso, A.M. Aguilera, D. Maldonado, M. Lanza, Time series statistical analysis: a powerful tool to evaluate the variability of resistive switching memories, J. Appl. Phys. 125 (2019), 174504.

[32] P. Huang, X.Y. Liu, B. Chen, H.T. Li, Y.J. Wang, Y.X. Deng, J.F. Kang, A physicsbased compact model of metal-oxide-based RRAM DC and AC operations, IEEE Trans. Electron. Devices 60 (12) (2013) 4090-4097.

[33] X. Guan, S. Yu, H.-S. Philip Wong, A SPICE compact model of metal oxide resistive switching memory with variations, Electron Device Letters, IEEE 33 (10) (2012) 1405-1407.

[34] P. Chen, S. Yu, Compact modeling of RRAM devices and its applications in 1T1R and 1S1R Array design, IEEE Trans. Electron. Devices 62 (12) (2015) 4022-4028.

[35] M. Bocquet, D. Deleruyelle, H. Aziza, C. Muller, J.-M. Portal, T. Cabout, E. Jalaguier, Robust compact model for bipolar oxide-based resistive switching memories, IEEE Trans. Electron Devices 61 (2014) 674-681.

[36] Z. Jiang, Y. Wu, S. Yu, L. Yang, K. Song, Z. Karim, H.-S.P. Wong, A compact model for metal-oxide resistive random access memory with experiment verification, IEEE Trans. Electron. Devices 63 (5) (2016) 1884-1892.

[37] P. Huang, D. Zhu, S. Chen, Z. Zhou, Z. Chen, B. Gao, J. Kang, Compact model of HfOX-based electronic synaptic devices for neuromorphic computing, IEEE Trans. Electron. Devices 64 (2) (2017) 614-621.

[38] J.B. Roldán, G. González-Cordero, R. Picos, E. Miranda, F. Palumbo, F. Jiménez Molinos, E. Moreno, D. Maldonado, S.B. Baldomá, M. Moner Al Chawa, C. de Benito, S.G. Stavrinides, J. Suñé, L.O. Chua, On the thermal models for resistive random access memory circuit simulation, Nanomaterials 11 (2021) 1261. 
[39] S. Aldana, P. García-Fernández, R. Romero-Zaliz, M.B. González, F. JiménezMolinos, F. Gómez-Campos, F. Campabadal, J.B. Roldán, Resistive switching in $\mathrm{HfO}_{2}$ based valence change memories, a comprehensive 3D kinetic Monte Carlo approach, J. Phys. D. Appl. Phys. 53 (2020), 225106.

[40] S. Dirkmann, J. Kaiser, C. Wenger, T. Mussenbrock, Filament growth and resistive switching in hafnium oxide Memristive devices, ACS Appl. Mater. Interfaces 10 (17) (2018) 14857-14868.

[41] S. Aldana, E. Pérez, F. Jimenez-Molinos, C. Wenger, J.B. Roldán, Kinetic Monte Carlo analysis of data retention in $\mathrm{Al}: \mathrm{HfO}_{2}$-based resistive random access memories, Semicond. Sci. Technol. 35 (2020), 115012.

[42] A. Padovani, L. Larcher, O. Pirrotta, L. Vandelli, G. Bersuker, I.E.E.E. Member, Microscopic modeling of $\mathrm{HfO}_{\mathrm{x}}$ RRAM operations: from forming to switching, IEEE Trans. Electron. Devices 62 (6) (2015) 1998-2006.

[43] S. Menzel, P. Kaupmann, R. Waser, Understanding filamentary growth in electrochemical metallization memory cells using kinetic Monte Carlo simulations, Nanoscale 7 (2015) 12673.

[44] M. von Witzleben, K. Fleck, C. Funck, B. Baumkötter, M. Zuric, A. Idt, T. Breuer, R. Waser, U. Böttger, S. Menzel, Investigation of the impact of high temperatures on the switching kinetics of redox-based resistive switching cells using a high-speed nanoheater, Adv. Electron. Mater. 3 (2017) 1700294, https://doi.org/10.1002/ aelm.201700294.

[45] S. Poblador, M. Maestro-Izquierdo, M. Zabala, M.B. González, F. Campabadal, Methodology for the characterization and observation of lamentary spots in HfOxbased memristor devices, Microelectron. Eng. 223 (2020), 111232.

[46] J. Guy, G. Molas, P. Blaise, M. Bernard, A. Roule, G. Le Carval, V. Delaye, A. Toffoli, G. Ghibaudo, F. Clermidy, B. De Salvo, L. Perniola, Investigation of forming, SET, and data retention of conductive-bridge random-access memory for stack optimization, IEEE Trans. Electron. Devices 62 (11) (2015) 3482-3489.
[47] M. Ibáñez, J. Roldán, A. Roldán, R. Yáñez, A comprehensive characterization of the threshold voltage extraction in MOSFETs transistors based on smoothing splines, Math. Comput. Simul. 102 (2014) 1-10.

[48] D. Maldonado, A.M. Roldán, M.B. González, F. Jiménez-Molinos, F. Campabadal, J. B. Roldán, Influence of magnetic field on the operation of TiN/Ti/ $\mathrm{HfO}_{2} / \mathrm{W}$ resistive memories, Microelectron. Eng. 215 (2019), 110983.

[49] P. González, M.J. Ibáñez, A.M. Roldán, J.B. Roldán, An in-depth study on WENObased techniques to improve parameter extraction procedures in MOSFET transistors, Math. Comput. Simul. 118 (2015) 248-257.

[50] D. Barrera, M.J. Ibáñez, A.M. Roldán, J.B. Roldán, R. Yáñez, Polynomial pattern finding in scattered data, J. Comput. Appl. Math. 318 (2017) 107-116.

[51] D. Maldonado, F. Aguirre, G. González-Cordero, A.M. Roldán, M.B. González, F. Jiménez-Molinos, F. Campabadal, E. Miranda, J.B. Roldán, Experimental study of the series resistance effect and its impact on the compact modeling of the conduction characteristics of $\mathrm{HfO}_{2}$-based resistive switching memories, J. Appl. Phys. 130 (2021), 054503.

[52] M.A. Villena, J.B. Roldán, F. Jiménez-Molinos, J. Suñé, S. Long, E. Miranda, M. Liu, A comprehensive analysis on progressive reset transitions in RRAMs, J. Phys. D. Appl. Phys. 7 (2014), 205102.

[53] C. Acal, J.E. Ruiz-Castro, A.M. Aguilera, F. Jiménez-Molinos, J.B. Roldán, Phasetype distributions for studying variability in resistive memories, J. Comput. Appl. Math. 345 (2019) 23-32.

[54] J.W. McPherson, Reliability Physics and Engineering. Time-to-Failure Modeling, Second ed., Springer, 2013.

[55] E. Pérez, D. Maldonado, C. Acal, J.E. Ruiz-Castro, F.J. Alonso, A.M. Aguilera, F. Jiménez-Molinos, Ch. Wenger, J.B. Roldán, Analysis of the statistics of device-todevice and cycle-to-cycle variability in TiN/Ti/Al:HfO $/$ /TiN RRAMs, Microelectron. Eng. 214 (2019) 104-109. 